\title{
An additional high oral loading dose of baricitinib improves clinical outcome in patients with COVID-19 pneumonia
}

\author{
Md. Jahidul Hasan ${ }^{1}$, Raihan Rabbani ${ }^{1}$, Ahmad Anam ${ }^{1}$, and Shihan Mahmud Huq ${ }^{1}$ \\ ${ }^{1}$ Square Group
}

July 27, 2020

\begin{abstract}
Baricitinib is a promising drug in COVID-19 pneumonia. The aim of this study is to compare the clinical outcome of moderateto-severe COVID-19 pneumonia treated with baricitinib with or without a loading dose. This prospective case-control study enrolled 37 adult patients where 17 patients (control) received baricitinib at $4 \mathrm{mg}$ oral daily dose and 20 patients (case) received an additional single $8 \mathrm{mg}$ oral loading dose. The median day to gain blood oxygen saturation level [?] 95\% (in room air) and return in normal breathing function were lower in case group than the control group $[29.4 \%(\mathrm{n}=17) / 10 \%(\mathrm{n}=20), \mathrm{P}<$ $0.05 ; 11.8 \%(\mathrm{n}=17) / 5 \%(\mathrm{n}=20), \mathrm{P}>0.05)$, respectively]. The requirement of intensive care unit and mechanical ventilation support were higher in control group than the case group. Thus, an additional loading dose of baricitinib revealed better clinical outcome in COVID-19 pneumonia.
\end{abstract}

\section{INTRODUCTION}

The coronavirus disease 2019 (COVID-19) caused by severe acute respiratory syndrome coronavirus 2 (SARSCoV-2), first introduces to world as an outbreak in Wuhan, China, in December 2019, was declared as a "global pandemic" by World Health Organization (WHO) on March 11, 2020. ${ }^{1}$ As of July 11, 2020, a total of 12322395 confirmed COVID-19 cases and 556335 related deaths have been reported worldwide. ${ }^{2}$ Acute respiratory distress syndrome (ARDS) due to hyperinflammation in the lungs and ultimately, respiratory failure from COVID-19 pneumonia has been reported as the prime cause of COVID-19-associated mortality ${ }^{3,4}$ ranging from $1 \%$ to more than $7 \%{ }^{5}$ Cytokine storm due to the over production of proinflammatory cytokines, including IL (interleukin)-6, IL-7, IL-8, etc. in critically ill patients with COVID-19 is a critical phase of the disease that may lead to acute respiratory failure or multiple organ dysfunction. ${ }^{6,7}$ Therefore, early detection of the COVID-19 infection and prompt management to suppress the cytokine storm is equally important in the patients with COVID-19. ${ }^{8}$

IL-6 plays a pivotal role in exacerbating the cytokine storm as a part of host's innate immune response mechanism which is mostly activated by IL-1 beta, tumor necrosis factor (TNF-alfa), Toll-like receptors (TLRs), prostaglandins, adipokines and other cytokines. ${ }^{9}$ High levels of IL-6 and IL- 8 were detected in patients with severe SARS-CoV-1 infection, where high-magnitude of innate inflammatory response in correspondence to SARS-CoV-1 invasion in the host's respiratory tract was extensively induced by IL-6. ${ }^{10}$ Similarly, high plasma level of IL-6 along with other proinflammatory cytokines, such as IL-2, IL-7, IL-8, IL-10, macrophage inflammatory protein (MIP1A), monocyte chemoattractant protein (MCP1) and TNF-alfa have been found in critically ill patients with COVID-19 and these cytokines significantly contribute in the severity of the disease. ${ }^{5,6,8}$

Receptor-mediated endocytosis is a process through which most viruses penetrate into the host cells. AP2associated protein kinase 1 (AAK1) is a regulator of this endocytosis and inactivation of this enzyme interrupt 
the entry of the virus into the host cells. ${ }^{11}$ Baricitinib, a janus kinase (JAK) inhibitor, is an approved drug for rheumatoid arthritis (RA). In effect, baricitinib specifically prevents the expression of proinflammatory cytokines, including IL-6 by inhibiting JAK. Interestingly, among the six highly potent inhibitor of AAK1, baricitinib is one of them which works by binding with the cyclin G-associated kinase that disrupt endocytosis, competently leading to reduction in viral load. ${ }^{11,12}$ Nowadays, baricitinib at a $4 \mathrm{mg}$ once daily oral dose has been highlighted as a promising investigational anti-inflammatory (IL-6 inhibitor) drug therapy in patients with COVID-19 pneumonia. ${ }^{4,11,12,14}$ The pharmacokinetics, pharmacodynamics and safety data of baricitinib at a $4 \mathrm{mg}$ daily dose in COVID-19 are still under investigation and to date, no standard dosing of baricitinib in COVID-19 has yet been established worldwide. ${ }^{11-15}$ The objective of this study was to compare the clinical outcomes of patients with moderate-to-severe COVID-19 pneumonia treated with baricitinib at a $4 \mathrm{mg}$ once daily oral dose with or without a high oral loading dose.

\section{MATERIALS AND METHODS}

\section{Study design and data collection}

This prospective case-control study was conducted in the "COVID-19 Unit" of Square hospital ltd., Dhaka, Bangladesh on $37(\mathrm{~N})$ adult ([?] 18 years) patients with moderate to severe COVID-19 symptoms admitted to this hospital from May 15-June 13, 2020. All the patients were Bangladeshi citizen and older than 18 years. Dexamethasone (corticosteroid) (10-20 mg/day, intravenously) and baricitinib combinely was started on day one of admission for down-regulating the inflammatory processes in lungs. Seventeen (n) patients in "no loading dose (LND) group" (control) received baricitinib $4 \mathrm{mg}$ daily orally for 2 weeks, where as 20 (n) patients in "loading dose (LD) group" (case) received baricitinib $8 \mathrm{mg}$ single dose orally as a loading dose on day one and then, $4 \mathrm{mg}$ daily orally from day 2 to 14 . The dose of baricitinib was taken on empty stomach. Simple random sampling method was used in this study. The local brand "Baritor 2" (baricitinib $2 \mathrm{mg}$ tablet) of Square pharmaceuticals ltd., Bangladesh was used in this study. Data of this study were collected from online patient-wise data archive of this hospital and directly from patient-prescriptions on daily basis by participating doctors in COVID-19 unit. Adverse events were routinely monitored and reported by two clinical pharmacists.

\section{Sample inclusion criteria were:}

1. presence of SARS-CoV 2 in the nasal/oral swabs

2. no previous history of COVID-19 infection

3. having at least 3 of the following symptoms: fever, cough, tiredness, sore throat, anosmia, respiratory distress, and myalgia

4. evidence of pneumonia in radiological diagnosis

\section{Sample exclusion criteria were:}

1. more than 10 days from onset of symptoms

2. patient with pregnancy

3. any history of trauma or surgical procedure within the last 3 months of admission

4. any history of acute/chronic autoimmune disease

5. evidence of bacterial or fungal coinfection

\section{Definition of moderate and severe COVID-19 pneumonia:}

Radiological evidence of bilateral pneumonia with clinical signs (fever, cough, difficulty breathing, tachypnea), no sign of severe stage of pneumonia, $\mathrm{SpO}_{2}$ (peripheral capillary blood oxygen saturation level) [?] $90 \%$ on room air (RA), ratio of arterial oxygen partial pressure to fractional inspired oxygen $\left(\mathrm{PaO}_{2} / \mathrm{FiO}_{2}\right)$ 100$300 \mathrm{mmHg}$; radiological evidence of severe pneumonia with clinical signs (fever, cough, difficulty breathing, tachypnea) with at least one of the following signs: respiratory rate $>30$ breaths/min, severe respiratory distress, and $\mathrm{SpO}_{2}<90 \%$ on room air. ${ }^{16} \mathrm{SpO}_{2}$ [?] $95 \%$ was considered as the targeted $\mathrm{SpO}_{2}$ in patients in this study. The severity of the disease and the progression of the symptoms of COVID-19 were measured 
on daily basis by assessing physical condition of the patients and the laboratory investigations, including hematological tests, tests for liver and kidney functions, inflammation and infection markers, and arterial blood gas test.

\section{Data analysis and ethical approval}

Data were analyzed with SPSS version 22.0 statistical software (SPSS, Chicago, IL, USA). Descriptive statistics were presented through median value and interquartile range (IQR). Categorical variables were compared using Fisher's exact test, and continuous variables were compared using Mann-Whitney $U$ test. $P$ values [?] 0.05 were considered statistically significant. The Square Hospital Ethical Committee granted ethical approval (no. 2004SH-OR024) for this study on April 11, 2020. All patients gave their written consent to participate to the study.

\section{RESULTS}

The number of male patients in both the groups was higher than the number of female patients with a median age of 52 (50.5-62) and 59 (49.8-69) in NLD and LD group, respectively $(P=0.414)$. Clinical characteristics, including blood-oxygen saturation profile, respiratory and cardiac functions, hematological components, and infection markers of all the patients $(\mathrm{N}=37)$ in the two groups are shown in Table 1 and compared. On admission, symptoms of moderate/severe COVID-19 pneumonia and predisposed comorbidities in the two groups were similar.

All patients $(\mathrm{N}=37)$ in both the groups tolerated baricitinib therapy well with no mild-to-serious adverse events (AEs) during the study period. No bacterial or fungal or any other opportunistic infections, hepatic or hematological toxicity were observed in the groups.

The median day to reach the targeted $\mathrm{SpO}_{2}$ ([?]95\% in RA) was less in the LD group (case) [3 (IQR: 2-8)] than that in the NLD group (control) [4 (IQR: 4-5)] $(P=0.180)$, and the median day to return in normal breathing function was significantly less in the LD group [5 (IQR: 4-5)] than that in the NLD group [8 (IQR: 7-10)]. ICU support was required higher in the NLD group with more requirement of mechanical ventilation support than that in the LD group $[29.4 \%(\mathrm{n}=17) / 10 \%(\mathrm{n}=20), P<0.05 ; 11.8 \%(\mathrm{n}=17) / 5 \%(\mathrm{n}=20)$, $P=0.141$, respectively] (Table 2). The median day of hospitalization was lower in the LD group [12 (IQR: 10-14)] than that in the NLD group [15 (IQR: 9-18.5)] and this was statistically significant. The 30-day all cause mortality rate was similar in the two groups.

\section{DISCUSSION}

This study found that a single $8 \mathrm{mg}$ oral loading dose of baricitinib added to its daily $4 \mathrm{mg}$ maintenance dose for up to 2 weeks exhibited better clinical outcomes in patients with moderate-to-severe COVID-19 than 4 mg daily oral dose regimen without having a loading dose. The targeted $\mathrm{SpO}_{2}$ ([?]95\%) was gained earlier in patients received an early $8 \mathrm{mg}$ of loading dose of baricitinib than the patients treated without a loading dose of baricitinib. Similarly, the requirement of supplement oxygen support was significantly reduced earlier in patients treated with an extra $8 \mathrm{mg}$ of loading dose of baricitinib that the patients received only $4 \mathrm{mg}$ daily maintenance dose.

Study showed that around $80 \%$ of patients with COVID-19 develop acute lung inflammation due to massive cytokine storm ${ }^{3,5-7}$ resulting in hypoxemia, impaired hypoxic pulmonary vasoconstriction, and high altitude pulmonary edema leading to acute respiratory distress syndrome (ARDS $)^{3,8,17}$ which may result death in 2\%-7\% of patients with COVID-19 pneumonia ${ }^{18}$ Janus kinases, a tyrosine kinase, play its major antiinflammatory role in the cytokine signaling pathways by constitutively binding to cytokine receptors. To potentiate a cytokine storm, proinflammatory cytokines need to transmit more signals through signaling pathways. In-role, more than 40 different types of cytokines transmit signals through JAKs, including JAK1, JAK2, JAK3, and tyrosine kinase-2 (TyK2). ${ }^{13,18}$ Baricitinib has an oral bioavailability of approximately $79 \%$, plasma protein binding ability up to $50 \%$, mean half-life of approximately $12.5 \mathrm{~h}$ (in RA), and shows serum drug level of approximately $100-300 \mathrm{nM}$ following a $4 \mathrm{mg}$ oral dose. ${ }^{13,19}$ The low molecular weight baricitinib concentration-dependently inhibits JAK1 and JAK2 with high target-specificity. ${ }^{13}$ 
In addition, baricitinib suppresses the production of Type-I interferons (IFNs) from plasmacytoid dendritic cells (pDCs), inhibits the synthesis of interleukin (IL)-6 from B cells ${ }^{20}$ and possesses strong anti-viral property by inhibiting endocytosis. ${ }^{21}$ Therefore, down-regulation of signal transmission of proinflammatory cytokines through JAKs, and suppression of other intrinsic inflammatory pathways makes baricitinib a suitable anti-inflammatory drug therapy in the treatment of COVID-19 pneumonia. The additional strong anti-viral property of baricitinib along with its selective anti-inflammatory ability has attributed its superiority to other oral investigational anti-inflammatory drugs in the treatment of COVID-19 pneumonia.

The plasma drug concentration of baricitinib at 2-4 $\mathrm{mg}$ daily oral dose is sufficient to exhibit its antiinflammatory roll (JAKs inhibition) in $\mathrm{RA}^{11,12}$ but, a standard dose of baricitinib to achieve its optimum therapeutic outcome in COVID-19 pneumonia is still under investigation. In a recent study with a small sample size showed an improved clinical outcome of patients with COVID-19 with baricitinib at $4 \mathrm{mg}$ per day of oral dose for 2 weeks. ${ }^{14}$ In this study, a 2-fold higher $(2 \times 4 \mathrm{mg})$ single loading dose of baricitinib followed by $4 \mathrm{mg}$ daily dose showed better therapeutic response in the management of moderate-to-severe COVID-19 pneumonia, and a superior clinical outcome was attributed in patients (case group) within 2 weeks of therapy with reduced hospitalization time and mortality rate. The major limitation of this study were small study sample size, short duration of study, no proven justification of using $8 \mathrm{mg}$ loading dose of baricitinib, and unavailability of serum cytokines monitoring facility in the study setup.

\section{CONCLUSION}

Severe pneumonia associated with COVID-19 is a major cause of mortality in COVID-19 infection. No anti-inflammatory drug, including baricitinib for the treatment of COVID-19 pneumonia has yet been recommended. In this study, 2-week-long $4 \mathrm{mg}$ once daily oral maintenance therapy of baricitinib following a single $8 \mathrm{mg}$ loading dose in moderate-to-severe COVID-19 pneumonia showed faster return to normal respiratory function with reduced requirement of ICU and ventilator support, length of hospital stay, and mortality rate than a loading dose-less $4 \mathrm{mg}$ once daily oral dose of baricitinib.

\section{ACKNOWLEDGEMENTS}

The authors of this study are very grateful to the authority of the Square hospital for their ethical permission for this study and also grateful to the participated patients for their consent for this study.

\section{CONTRIBUTIONS}

MJH: conceptualization, study design, formal data analysis, conducted the study, developed the analytical assay, analyzed the samples, prepared the results, manuscript writing, critical review, and editing

RR: supervise the study, critical review, and editing

AMA: formal data analysis, conducted the study, critical review, and editing

SMRH: conducted the study, analyzed the samples, critical review, and editing

\section{COMPETING INTERESTS}

The authors declare that there is no conflict of interest.

\section{FUNDING SOURCE}

No external funding source for this study.

\section{DATA SHARING}

The data that support the findings of this study are available from the corresponding author upon reasonable request.

\section{ORCID ID}

Md Jahidul Hasan: https://orcid.org/0000-0001-7038-6437 


\section{REFERENCES}

1. World Health Organization. Coronavirus disease (COVID-19) pandemic. 2020. https://www.euro.who.int/en/health-topics/health-emergencies/coronavirus-covid-19/novelcoronavirus-2019-ncov. Accessed 10 July, 2020.

2. World Health Organization. Coronavirus disease (COVID-2019) situation reports-173. Geneva: WHO; 2020. https://www.who.int/docs/default-source/coronaviruse/situation-reports/20200711covid-19-sitrep-173.pdf?sfvrsn=949920b4_2. Accessed 11 July, 2020.

3. Ruan Q, Yang K, Wang W, Jiang L, Song J. Clinical predictors of mortality due to COVID-19 based on an analysis of data of 150 patients from Wuhan, China. Intensive Care Med . 2020;46(5):846-848.

4. Atal S, Fatima Z. IL-6 Inhibitors in the Treatment of Serious COVID-19: A Promising Therapy? Pharmaceut Med . 2020:1-9.

5. Onder G, Rezza G, Brusaferro S. Case-fatality rate and characteristics of patients dying in relation to COVID-19 in Italy [JAMA Network]. https ://jaman etwor k.com/journ als/jama/fulla rticl e/27636 67. Accessed 29 April, 2020.

6. Chen N, Zhou M, Dong X, et al. Epidemiological and clinical characteristics of 99 cases of 2019 novel coronavirus pneumonia in Wuhan, China: a descriptive study. Lancet . 2020;395(10223):507-513.

7. Huang C, Wang Y, Li X, et al. Clinical features of patients infected with 2019 novel coronavirus in Wuhan, China. Lancet . 2020;395(10223):497-506.

8. Luo P, Liu Y, Qiu L, Liu X, Liu D, Li J. Tocilizumab treatment in COVID-19: A single center experience. J Med Virol . 2020;92(7):814-818.

9. Hunter CA, Jones SA. IL-6 as a keystone cytokine in health and disease. Nat Immunol . 2015;16(5):448457.

10. Wang WK, Chen SY, Liu IJ, et al. Severe Acute Respiratory Syndrome Research Group of the National Taiwan University College of Medicine/NTU Hospital. Temporal relationship of viral load, ribavirin, interleukin (IL)-6, IL-8, and clinical progression in patients with severe acute respiratory syndrome. Clin Infect Dis . 2004;39(7):1071-1075.

11. Richardson P, Griffin I, Tucker C, et al. Baricitinib as potential treatment for 2019-nCoV acute respiratory disease. Lancet . 2020;395(10223):e30-e31.

12. Cingolani A, Tummolo AM, Montemurro G, et al. Baricitinib as rescue therapy in a patient with COVID-19 with no complete response to sarilumab. Infection . 2020:1-5.

13. Kubo S, Nakayamada S, Sakata K, et al. Janus kinase inhibitor baricitinib modulates human innate and adaptive immune system.Front Immunol . 2018;9:1510.

14. Cantini F, Niccoli L, Matarrese D, Nicastri E, Stobbione P, Goletti D. Baricitinib therapy in COVID-19: A pilot study on safety and clinical impact. J Infect . 2020:S0163-4453(20)30228-0.

15. Bronte V, Ugel S, Tinazzi E, et al. Baricitinib restrains the immune dysregulation in COVID-19 patients. 2020. https://doi.org/10.1101/2020.06.26.20135319

16. World Health Organization. Clinical management of COVID-19. Interim guidance. 2020. https://www.who.int/publications/i/item/clinical-management-of-covid-19. Accessed 21 July, 2020.

17. Archer SL, Sharp WW, Weir EK. Differentiating COVID-19 pneumonia from acute respiratory distress syndrome and high altitude pulmonary edema. Circulation . 2020;142(2):101-104.

18. Karaman MW, Herrgard S, Treiber DK, et al. A quantitative analysis of kinase inhibitor selectivity. Nat Biotechnol . 2008;26(1):127-132.

19. Kim H, Brooks KM, Tang CC, et al. Pharmacokinetics, pharmacodynamics, and proposed dosing of the oral JAK1 and JAK2 inhibitor baricitinib in pediatric and young adult CANDLE and SAVI Patients. Clin Pharmacol Ther . 2018;104(2):364-373.

20. McInnes IB, Byers NL, Higgs RE, et al. Comparison of baricitinib, upadacitinib, and tofacitinib mediated regulation of cytokine signaling in human leukocyte subpopulations. Arthritis Res Ther . 2019;21(1):183.

21. Perricone C, Triggianese P, Bartoloni E, et al. The anti-viral facet of anti-rheumatic drugs: Lessons from COVID-19. J Autoimmun . 2020;111:102468. 
Table 1: Baseline demographic, symptoms of COVID-19, comorbidity and laboratory findings in patients treated with or without a loading dose of baricitinib

\begin{tabular}{|c|c|c|c|}
\hline Characteristics & NLD group $(\mathrm{N}=17)$ & LD group $(\mathrm{N}=20)$ & $P$ vi \\
\hline Male/female, n (\%) & $13 / 4(76 / 24)$ & $16 / 4(80 / 20)$ & 0.61 \\
\hline Age (year), median (IQR) & $52(50.5-62)$ & $59(49.8-69)$ & 0.41 \\
\hline Days from onset of symptoms-to-hospitalization, median (IQR) & $7(4.5-7.5)$ & $6.5(5-7)$ & 0.18 \\
\hline Fever $\left({ }^{\circ} \mathrm{F}\right)$, median $(\mathrm{IQR})$ & $101(100-101.5)$ & $100.5(100-101.75)$ & 0.91 \\
\hline Dry cough, n $(\%)$ & $17(100)$ & $20(100)$ & 0.00 \\
\hline Shortness of breath, n (\%) & $14(82.4)$ & $16(80)$ & 0.8 \\
\hline Weakness, n (\%) & $17(100)$ & $20(100)$ & 0.00 \\
\hline Diarrhea, n (\%) & $3(17.6)$ & $5(25)$ & 0.2 \\
\hline Anosmia, n (\%) & $11(64.7)$ & $14(70)$ & 0.51 \\
\hline Sore throat, $\mathrm{n}(\%)$ & $9(52.9)$ & $12(60)$ & 0.02 \\
\hline Diabetes, n (\%) & $14(82.4)$ & $17(85)$ & 0.67 \\
\hline Hypertension, $\mathrm{n}(\%)$ & $13(76.5)$ & $12(60)$ & 0.0 \\
\hline CVD, n $(\%)$ & $6(35.3)$ & $4(20)$ & 0.05 \\
\hline Bronchial asthma, n (\%) & $4(23.5)$ & $6(30)$ & $0.3 \xi$ \\
\hline CKD, n $(\%)$ & $2(11.8)$ & $3(15)$ & 0.5 \\
\hline COPD, n $(\%)$ & $2(11.8)$ & $3(15)$ & 0.5 \\
\hline Severe obesity, n (\%) & $2(11.8)$ & $2(10)$ & 0.7 \\
\hline Malignancy, n (\%) & $1(5.9)$ & $3(15)$ & $0.0^{\prime}$ \\
\hline $\mathrm{PD}, \mathrm{n}(\%)$ & $0(0)$ & $2(10)$ & 0.00 \\
\hline $\mathrm{SpO}_{2}(\%)$, median (IQR) & $89(87.5-90)$ & $89.5(88-90)$ & 0.7 \\
\hline Respiratory rate, (breaths/min), median (IQR) & $22(20.5-24)$ & $23(21-24)$ & 0.8 \\
\hline $\mathrm{PaO}_{2} / \mathrm{FiO}_{2}(\mathrm{mmHg})$, median (IQR) & $240(195.5-263.5)$ & $245(193.8-262.3)$ & 0.0 \\
\hline Heart rate (beat/min), median (IQR) & $98(85.5-105.5)$ & $94.5(84.5-113)$ & 0.1 \\
\hline CRP (mg/L), median (IQR) & $43.5(30-220)$ & $77.1(40.9-211.5)$ & 0.9 \\
\hline Procalcitonin (ng/mL), median (IQR) & $0.12(0.06-0.49)$ & $0.18(0.07-0.86)$ & 0.0 \\
\hline WBC $(\mathrm{K} / \mu \mathrm{L})$, median $(\mathrm{IQR})$ & $6.3(4.7-10)$ & $8(6.3-14.3)$ & 0.0 \\
\hline Neutrophils (\%), median (IQR) & $87.6(79.4-92.1)$ & $82.5(76.8-88.1)$ & 0.6 \\
\hline Lymphocytes (\%), median (IQR) & $13.9(11.9-15.8)$ & $12.2(8.2-14.5)$ & 0.0 \\
\hline Platelet $(\mathrm{K} / \mu \mathrm{L})$, median $(\mathrm{IQR})$ & $186(128-255)$ & $175(133.3-245.3)$ & 0.6 \\
\hline D-Dimer (mg /L FEU), median (IQR) & $2.7(1.7-7.6)$ & $2(1.5-3.2)$ & 0.0 \\
\hline Serum Ferritin $(\mathrm{ng} / \mathrm{mL})$, median (IQR) & $434(293.5-543.5)$ & $470(361.3-604.8)$ & 0.2 \\
\hline LDH ((U/L), median (IQR) & $272(216-404.5)$ & $334(295-390.5)$ & 0.0 \\
\hline Creatinine $(\mathrm{mg} / \mathrm{dL})$, median (IQR) & $0.9(0.8-1)$ & $0.9(0.7-1.1)$ & 0.2 \\
\hline $\operatorname{ALT}(\mathrm{U} / \mathrm{L})$, median (IQR) & $34(29-57)$ & $44(35-54)$ & 0.1 \\
\hline $\operatorname{AST}(\mathrm{U} / \mathrm{L})$, median (IQR) & $35(25.5-49)$ & $40(29.5-58.8)$ & 0.3 \\
\hline MEWS, median (IQR) & $3(2-3)$ & $3(2-3)$ & 0.4 \\
\hline
\end{tabular}

$\mathrm{IQR}=$ interquartile range; $\mathrm{n}=$ number; $\%=$ percentage $;{ }^{\circ} \mathrm{F}=$ grade Fahrenheit CVD $=$ cardiovascular disease; $\mathrm{CKD}=$ chronic kidney disease; $\mathrm{COPD}=$ chronic obstructive pulmonary disease; $\mathrm{PD}=$ parkinson's disease; $\mathrm{SpO}_{2}=$ peripheral capillary oxygen saturation; min = minute; $\mathrm{PaO}_{2} / \mathrm{FiO}_{2}=$ ratio of arterial oxygen partial pressure to fractional inspired oxygen; $\mathrm{mmHg}=$ millimetre of mercury; $\mathrm{CRP}=\mathrm{C}$-reactive protein; $\mathrm{mg}=$ milligram; $\mathrm{L}=$ liter; FEU $=$ fibrinogen equivalent units; $\mathrm{ng}=$ nanogram; $\mathrm{WBC}=$ white blood cells; $\mathrm{K} / \mu \mathrm{L}=$ thousand cells per micro liter; $\mathrm{LDH}=$ lactate dehydrogenase; $\mathrm{U} / \mathrm{L}=$ units per liter; $\mathrm{dL}=$ deciliter; ALT $=$ alanine aminotransferase; AST $=$ aspartate aminotransferase; MEWS $=$ Modified Early Warning Score.

Table 2: Clinical outcomes in patients with COVID-19 treated with baricitinib with or without 


\section{a loading dose}

\begin{tabular}{llll}
\hline Parameters & NLD group (n= 17) & LD group (n= 20) & 1 \\
\hline Days required to increase $\mathrm{SpO}_{2}[?] 95 \%$ in RA, median (IQR) & $4(4-5)$ & $3(2-8)$ & 0 \\
Days required to stop the need of supplement oxygen, median (IQR) & $8(7-10)$ & $5(4-5)$ & 0 \\
Patients required ICU support, n (\%) & $5(29.4)$ & $2(10)$ & 0 \\
Patients intubated on ventilator, n (\%) & $2(11.8)$ & $1(5)$ & 0 \\
Length of hospital stay (day), median (IQR) & $15(9-18.5)$ & $12(10-14)$ & 0 \\
30-day mortality, n (\%) & $1(5.9)$ & $1(5)$ & 0 \\
\hline
\end{tabular}

$\mathrm{NLD}=$ no loading dose; $\mathrm{LD}=$ loading dose $\mathrm{SpO}_{2}=$ peripheral capillary oxygen saturation; $\mathrm{RA}=$ rheumatoid arthritis; IQR $=$ interquartile range; ICU $=$ intensive care unit; $\mathrm{n}=$ number; $\%=$ percentage.

\section{Hosted file}

Table 1.docx available at https://authorea.com/users/346533/articles/472490-an-additionalhigh-oral-loading-dose-of-baricitinib-improves-clinical-outcome-in-patients-with-covid19-pneumonia

\section{Hosted file}

Table 2.docx available at https://authorea.com/users/346533/articles/472490-an-additionalhigh-oral-loading-dose-of-baricitinib-improves-clinical-outcome-in-patients-with-covid19-pneumonia 